\title{
Les singularités thermiques d'un espace périurbain dans une agglomération de dimension moyenne : le cas de Caen durant l'hiver 2018/2019
}

\author{
Olivier Cantat* et Alexandre Follin \\ Normandie Université, UNICAEN, CNRS, LETG, Caen, France
}

\begin{abstract}
Résumé - L'analyse comparative des données météorologiques de la station rurale de Carpiquet avec une station expérimentale en périphérie de la ville de Caen a permis de montrer la présence en hiver d'un léger îlot de chaleur péri-urbain (ICPU) (moyenne de $+0,4^{\circ} \mathrm{C}$ ). Il est caractérisé par un maximum d'intensité en fin d'après-midi et début de soirée $\left(+0,8^{\circ} \mathrm{C}\right)$ et des écarts faibles le reste du temps. Derrière cette image moyenne, une analyse du continuum temporel au pas de temps journalier puis horaire a mis en exergue des comportements différenciés selon les types de temps. Le caractère le plus original des résultats est la présence certes rare, mais significative en termes d'intensité, d'un îlot de fraîcheur péri-urbain (IFPU). Il se forme de nuit par temps clair et calme et se prolonge dans la matinée, jusqu'à présenter un maximum d'intensité vers $9 \mathrm{~h}$ UTC. Cette inversion par rapport au schéma classique de fonctionnement des îlots de chaleur urbain (ICU) s'explique par un mode d'occupation du sol favorable à la formation d'une poche froide par rayonnement nocturne, favorisée par l'absence totale de vent (effet de barrage du bâti et de la végétation) et par la faible densité urbaine, alors que sur la campagne ces mécanismes de refroidissement sont atténués par la persistance d'un léger flux. Cet IFPU résiste et s'intensifie en début de matinée, jusqu'à temps que le soleil et la turbulence de l'air ne viennent échauffer et disperser cette pellicule d'air froid plus dense. Ici, les conditions stationnelles semblent donc primer sur les effets purement urbains car l'agglomération de Caen ne présente pas une masse suffisante pour créer une " bulle chaude » jusque dans ses quartiers périphériques quand un type de temps radiatif s'impose.
\end{abstract}

Mots-clés : topoclimat / îlot de chaleur urbain (ICU) / îlot de fraîcheur péri-urbain (IFPU) / types de temps / Caen.

\begin{abstract}
Thermal singularities of a peri-urban area in a medium-sized conurbation: the case of Caen in the winter of 2018/2019. The comparative analysis of meteorological data from the rural station of Carpiquet with an experimental weather station on the outskirts of the city of Caen showed the presence in winter of a slight peri-urban heat island (PUHI) (average $+0.4^{\circ} \mathrm{C}$ ). It is characterized by a maximum intensity in late afternoon and early evening $\left(+0.8^{\circ} \mathrm{C}\right)$ and small differences the rest of the time. Behind this average image, an analysis of the temporal continuum at daily and then hourly time steps highlighted differentiated behaviours according to the weather types. The most original feature of the results is the rare but significant presence of a peri-urban freshness island (PUFI). It forms at night on clear and calm weather and extends into the morning, reaching a maximum intensity around 9:00 a.m. This inversion in relation to the classic urban heat island (UHI) operating pattern is explained by a land use pattern conducive to the formation of a cold pocket by night-time radiation, favoured by the total absence of wind (damming effect of buildings and vegetation) and by the low urban density, whereas in the countryside these cooling mechanisms are attenuated by the persistence of a slight wind. This IPFU resists and intensifies in the early morning, until the sun and air turbulence heat up and disperse this denser layer of cold air. Here, stationary conditions therefore seem to take precedence over purely urban effects, as the Caen conurbation does not have sufficient mass to create a "hot bubble" all the way to its outlying areas when a type of radiative weather is required.
\end{abstract}

Keywords: topoclimate / urban heat island (UHI) / peri-urban freshness island (PUFI) / weather types / Caen (Normandy).

\footnotetext{
* $\overline{\text { Auteur de correspondance }}$ : olivier.cantat@unicaen.fr
} 


\section{Introduction}

Dans les grandes agglomérations, l'extension de revêtements artificiels et la multiplication de constructions sont à l'origine d'une profonde modification du bilan local d'énergie. La formation d'un îlot de chaleur urbain (ICU) en est la manifestation la plus concrète, en lien avec la réduction des phénomènes évapotranspiratoires (déficit en eau des surfaces imperméabilisées et faible proportion d'espaces végétalisés), combiné au stockage de chaleur dans les matériaux de construction sous l'effet du soleil et de sa lente restitution de nuit (Dettwiller, 1970). Les caractères de l'ICU sont cependant très variables d'une agglomération à une autre, reflets de la multitude des combinaisons possibles entre les singularités géographiques et physionomiques de chaque site (contexte topographique, forme et densité du bâti, nature des matériaux...) et les spécificités du climat régional (fréquence des types de temps clairs et calmes favorables à l'expression des facteurs locaux). En conséquence, chaque agglomération possède sa propre «identité thermique », changeante dans l'espace comme dans le temps (Cantat, 2004).

Autrefois essentiellement objet d'étude de scientifiques et d'urbanistes, l'ICU est aujourd'hui fortement médiatisé et la population sensibilisée au phénomène, notamment en été pour se prémunir des désagréments provoqués par le caractère étouffant des nuits dans le cœur des villes en période de canicule $^{1}$, voire de la surmortalité associée quand ces conditions perdurent longtemps (INVS, 2003 ; Besancenot, 2004 ; IAU Île-de-France, 2010). Dans le cadre du réchauffement contemporain et des projections climatiques sur le $21^{\text {ème }}$ siècle, une attention toute particulière est donc portée à la connaissance du climat dans les très grandes métropoles mais quid de celles de taille moyenne? En France, à ce niveau scalaire, les références sont peu nombreuses: citons toutefois les travaux sur Nice (Carrega, 1992 et 2013), sur Strasbourg

\footnotetext{
${ }^{1}$ Le 9 août 2020, après une journée torride en Ile-deFrance (valeurs maximales de 36 à $38^{\circ} \mathrm{C}$ ), dans Paris intra-muros la température nocturne n'est pas descendue sous les $26^{\circ} \mathrm{C}$ (station de Lariboisière) alors qu'aux portes de la ville, dans l'espace aéré et végétalisé
}

(Kastendeuch et al., 2010), sur Nancy (Lauffenburger et al., 2014) ou bien encore sur Rennes et Dijon, avec le déploiement d'un véritable réseau d'observations (Dubreuil et al., 2011; Foissard, 2015 ; Richard et al., 2018 ; Foissard et al., 2019).

Dans le cadre d'une approche exploratoire sur le climat de l'agglomération caennaise (environ 200000 habitants), une station météorologique automatique a été placée dans un secteur pavillonnaire à la périphérie de la ville durant deux mois et demi, du $1^{\text {er }}$ janvier au 15 mars 2019. Les données collectées ont été comparées à celles de l'aéroport de Caen-Carpiquet, station Météo France située à environ $7 \mathrm{~km}$ du centre de la ville, représentative des conditions météorologiques régionales. L'étude a porté sur les températures, les autres paramètres météorologiques (notamment le vent et le rayonnement solaire) servant d'éléments explicatifs aux comportements thermiques variables entre la périphérie urbaine et la campagne environnante, dans leurs cycles journaliers et selon les types de temps.

Au-delà de la mise en évidence de l'îlot de chaleur urbain caennais (ICU) et de sa déclinaison périurbaine (ICPU), l'objet de cet article est de s'intéresser plus spécialement aux caractères de l'îlot de fraîcheur périurbain (IFPU) qui peut apparaitre de façon plus furtive mais significative, notamment en précisant ( $i$ ) l'intensité et les heures d'apparition privilégiées de ce phénomène, puis $\mathrm{d}$ 'envisager les facteurs et processus responsables de sa formation en dégageant (ii) les types de temps favorables à sa formation et en mettant en évidence (iii) les caractéristiques propres de cet espace urbain à l'échelle stationnelle. Ce comportement thermique original sera l'occasion de mettre en évidence le poids des facteurs géographiques de micro-échelle pouvant « perturber » le schéma classique de l'ICU, matérialisé à méso-échelle dans les grandes agglomérations par une décroissance progressive des températures nocturnes du centre-ville vers la campagne environnante.

au sud du Bois de Boulogne (station de Longchamp), on pouvait bénéficier d'une atmosphère agréable avec moins de $19^{\circ} \mathrm{C}$ au petit matin et plus de 7 heures consécutives sous $23^{\circ} \mathrm{C}$, seuil permettant une récupération physiologique correcte. 


\section{Présentation du secteur d'étude}

\subsection{Une agglomération de dimension moyenne}

Au recensement INSEE de 2016, l'agglomération caennaise constitue la $35^{\text {ème }}$ unité urbaine ${ }^{2}$ de France. Elle compte environ 200000 habitants, répartis sur une surface de $142 \mathrm{~km}^{2}$, soit une densité moyenne de l'ordre de $1400 \mathrm{hab} . / \mathrm{km}^{2}$. A l'échelle de la Normandie, c'est le $3^{\text {ème }}$ pôle urbain, loin derrière Rouen (au $12^{\text {ème }}$ rang national avec 467000 habitants sur $461 \mathrm{~km}^{2}$ ) mais assez proche dans ses dimensions du Havre (au $23^{\text {ème }}$ rang avec 235000 habitants sur $195 \mathrm{~km}^{2}$ ). A titre de comparaison, pour saisir les caractéristiques démographiques originales du secteur d'étude, rappelons que l'unité urbaine de Paris s'étale sur plus de $2800 \mathrm{~km}^{2}(\approx 20$ fois plus grand) et concentre plus de 10 millions d'habitants $(\approx 50$ fois plus peuplée).

\subsection{Un climat peu favorable à l'expression des facteurs géographiques locaux}

Dans la région de Caen, le développement de topoclimats pâtit d'un climat de type océanique (Pédelaborde, 1958 ; Escourrou, 1978), qualifié même d'océanique franc dans la typologie récente des climats en France de Joly et al. (2010). Ainsi, les conditions claires et calmes propices à l'établissement d'un climat local urbain sont bien souvent contrariées par la présence d'une couverture nuageuse durable et/ou d'un vent soutenu, éléments incompatibles avec la formation du dôme de chaleur au-dessus de la ville qui caractérise les grandes métropoles (Dettwiller, 1970). Ces caractères océaniques sont par ailleurs exacerbés du fait de la proximité de la mer $(15 \mathrm{~km}$ au Nord) et d'une topographie basse et ouverte (bas plateaux) ne prédisposant pas à la stagnation indispensable de l'air dans les basses couches.

En prenant comme critère une fraction journalière d'insolation supérieure ou égale à $66 \%$ et simultanément un vent moyen journalier inférieur

\footnotetext{
${ }^{2}$ "L'unité urbaine est une commune ou un ensemble de communes qui comporte sur son territoire une zone bâtie d'au moins 2000 habitants où aucune habitation n'est séparée de la plus proche de plus de 200 mètres. En outre, chaque commune concernée possède plus de la moitié de sa population dans cette zone bâtie » (source : site de l'INSEE).
}

à $3 \mathrm{~m} / \mathrm{s}$ (Cantat, 2004), les conditions nécessaires à la franche expression des facteurs géographiques locaux sont en moyenne présentes moins de $8 \%$ des jours de l'année à Caen (tableau 1). Ces temps clairs et calmes sont communément associés à la présence de hautes pressions atmosphériques $(68 \%$ des cas avec des valeurs supérieures à $1020 \mathrm{hPa}$ ), dans le cadre d'une situation synoptique largement dominée par une absence de flux marqué liée à des centres d'action sur l'Europe Centrale ${ }^{3}$ (40\%). Pardelà la moyenne annuelle, une nette saisonnalité se manifeste dans l'occurrence de ces conditions : elles sont logiquement beaucoup plus fréquentes en période estivale $(11,4 \%)$, quand les hauts géopotentiels subtropicaux remontent vers les latitudes moyennes et apportent le «beau temps », qu'en période hivernale $(4,3 \%)$, lorsque les perturbations atlantiques affectent régulièrement l'Europe de l'Ouest.

En résumé, les dimensions réduites de l'agglomération caennaise et les types de temps les plus fréquents ne sont pas favorables à l'imprégnation de la masse d'air par les différences de comportement énergétique des modes d'occupation du sol sur le bilan thermique dans les très basses couches de l'atmosphère (absorption d'énergie solaire par les matériaux de construction, accumulation puis restitution lente sous forme de chaleur sensible). Pour autant, ne peut-on voir apparaître des phénomènes météo-climatiques différenciant la ville de la campagne environnante ?

\section{Données et méthodes}

La référence météorologique officielle pour la région de Caen est fournie par les enregistrements de la station Météo France de Carpiquet, située à environ $7 \mathrm{~km}$ à l'ouest du centre de l'agglomération, sur le site de l'aéroport (route de Caumont). L'analyse comparative pour un secteur périurbain s'appuie sur les relevés d'une station météorologique

\footnotetext{
${ }^{3}$ Ce type de configuration à centre d'action sur l'Europe Centrale correspond à la présence d'une dorsale atlantique étendue sur l'Europe moyenne (63\% des cas) ou d'un anticyclone sur l'Europe Centrale (36 \% des cas).
} 
expérimentale placée en périphérie ouest de l'agglomération normande, allée des Merisiers, distante de $5 \mathrm{~km}$ de la station de référence « rurale » et de $2 \mathrm{~km}$ du centre-ville dense (figure 1).

Tableau 1. Combinaisons des éléments météorologiques favorables à l'expression des facteurs géographiques locaux sur les températures dans la région de Caen (Normale climatique 1981-2010). Sources : données Météo France de Caen-Carpiquet et grands types de circulation atmosphérique selon la classification de Hess \& Brezowsky (1952). Combinations of meteorological elements favourable to the expression of local geographical factors on temperatures in the Caen region (Climate normal period 1981-2010). Sources: Météo France data from Caen-Carpiquet and major atmospheric circulation patterns according to the classification of Hess \& Brezowsky (1952).
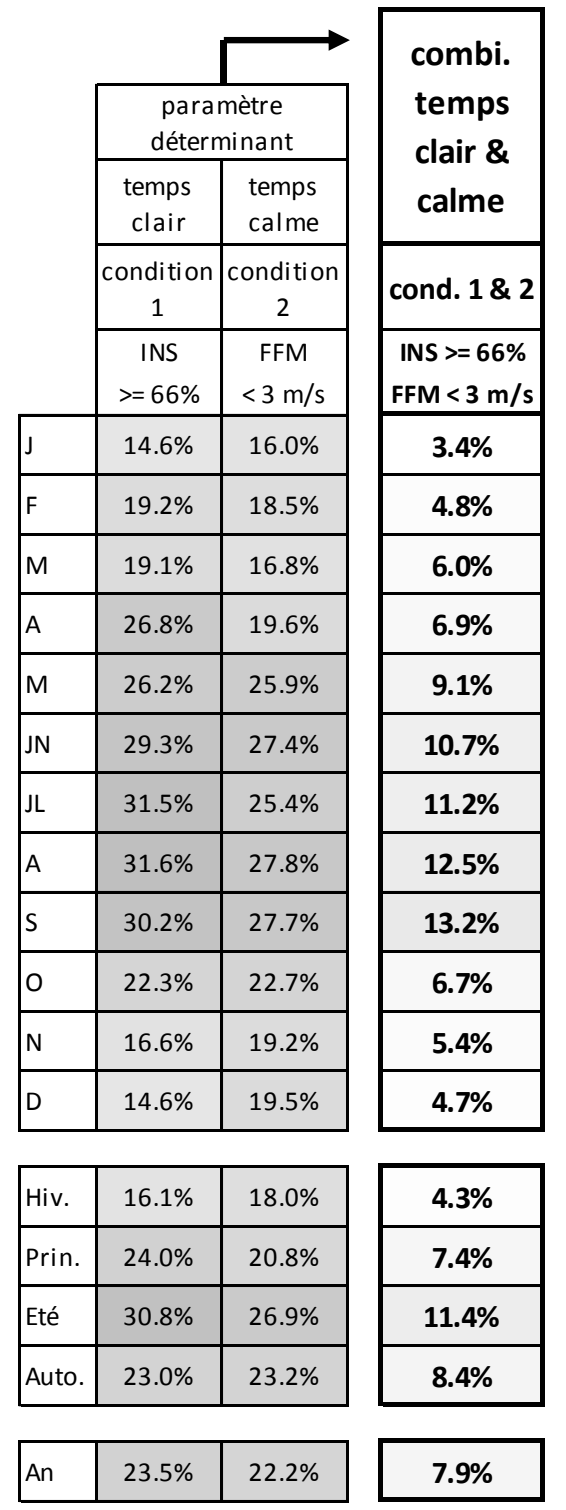

\begin{tabular}{|c|c|c|}
\hline \multicolumn{3}{|c|}{ Contexte barométrique } \\
associé au temps clair et calme
\end{tabular}

\begin{tabular}{|c|c|c|c|c|c|}
\hline \multicolumn{5}{|c|}{$\begin{array}{c}\text { Grand type de circulation atmosphérique } \\
\text { associé au temps clair et calme (classification } \\
\text { Hess \& Brezows ky en } 5 \text { GWT, Grosswettertypen) }\end{array}$} \\
\hline $\begin{array}{c}\text { CA } \\
\text { Europe } \\
\text { Centrale }\end{array}$ & $\begin{array}{c}\text { NW et } \\
\text { N }\end{array}$ & W & S & NE et E & indét. \\
\hline $41 \%$ & $25 \%$ & $13 \%$ & $16 \%$ & $3 \%$ & $3 \%$ \\
\hline $41 \%$ & $34 \%$ & $5 \%$ & $5 \%$ & $12 \%$ & $2 \%$ \\
\hline $46 \%$ & $20 \%$ & $13 \%$ & $14 \%$ & $5 \%$ & $2 \%$ \\
\hline $52 \%$ & $24 \%$ & $2 \%$ & $18 \%$ & $2 \%$ & $3 \%$ \\
\hline $46 \%$ & $25 \%$ & $5 \%$ & $13 \%$ & $12 \%$ & $0 \%$ \\
\hline $35 \%$ & $27 \%$ & $13 \%$ & $13 \%$ & $13 \%$ & $0 \%$ \\
\hline $39 \%$ & $27 \%$ & $14 \%$ & $5 \%$ & $14 \%$ & $0 \%$ \\
\hline $34 \%$ & $26 \%$ & $24 \%$ & $1 \%$ & $16 \%$ & $0 \%$ \\
\hline $34 \%$ & $28 \%$ & $22 \%$ & $13 \%$ & $4 \%$ & $0 \%$ \\
\hline $34 \%$ & $29 \%$ & $15 \%$ & $16 \%$ & $6 \%$ & $0 \%$ \\
\hline $37 \%$ & $45 \%$ & $8 \%$ & $6 \%$ & $4 \%$ & $0 \%$ \\
\hline $52 \%$ & $27 \%$ & $7 \%$ & $2 \%$ & $11 \%$ & $0 \%$ \\
\hline
\end{tabular}

\begin{tabular}{|c|c|c|}
\hline Hiv. & $16.1 \%$ & $18.0 \%$ \\
\hline Prin. & $24.0 \%$ & $20.8 \%$ \\
\hline Eté & $30.8 \%$ & $26.9 \%$ \\
\hline Auto. & $23.0 \%$ & $23.2 \%$ \\
\hline An & $23.5 \%$ & $22.2 \%$ \\
\hline
\end{tabular}

\begin{tabular}{|l|l|l|}
\hline $2 \%$ & $12 \%$ & $86 \%$ \\
\hline $4 \%$ & $33 \%$ & $63 \%$ \\
\hline $1 \%$ & $43 \%$ & $56 \%$ \\
\hline $2 \%$ & $20 \%$ & $78 \%$ \\
\hline \multicolumn{3}{|c|}{} \\
\hline $2 \%$ & $30 \%$ & $68 \%$ \\
\hline
\end{tabular}

\begin{tabular}{|l|c|c|c|c|c|}
\hline $45 \%$ & $29 \%$ & $8 \%$ & $7 \%$ & $9 \%$ & $2 \%$ \\
\hline $48 \%$ & $23 \%$ & $6 \%$ & $15 \%$ & $7 \%$ & $1 \%$ \\
\hline $36 \%$ & $27 \%$ & $17 \%$ & $6 \%$ & $14 \%$ & $0 \%$ \\
\hline $34 \%$ & $32 \%$ & $17 \%$ & $12 \%$ & $5 \%$ & $0 \%$ \\
\hline $40 \%$ & $27 \%$ & $13 \%$ & $10 \%$ & $9 \%$ & $1 \%$ \\
\hline
\end{tabular}

Un zoom à l'échelle locale et stationnelle permet de préciser le mode d'occupation du sol contrasté entre les deux espaces d'étude (figure 2). Carpiquet possède tous les attributs d'un site normalisé, matérialisé par un espace plan, dégagé et couvert d'herbe (Leroy, 1999). Pour la référence périurbaine, la station est installée également sur un site plan et couvert d'herbe mais elle est insérée dans un milieu pavillonnaire, caractérisé par une forte contrainte à la libre propagation des flux d'air et par la création d'effets d'ombre portée liés aux constructions. 


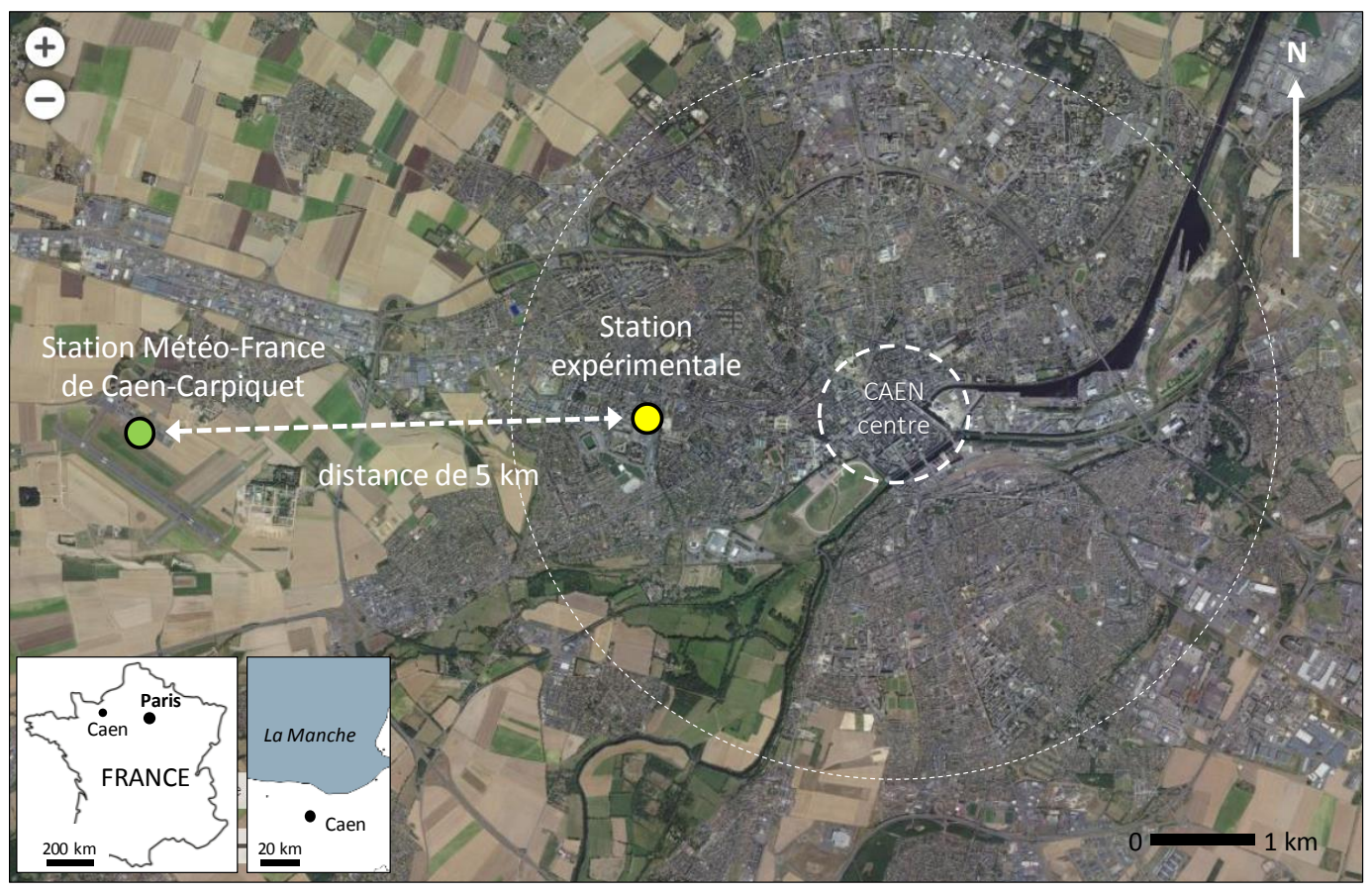

Figure 1. Localisation de la station météorologique référence de Caen-Carpiquet (rond vert) et de la station météorologique expérimentale en périphérie urbaine ouest de Caen, allée des Merisiers (rond jaune). Sources: données cartographiques Esri France, BRGM, complétées. Location of the reference weather station of Caen-Carpiquet (green circle) and the experimental weather station in the western urban periphery of Caen, allée des Merisiers (yellow circle). Source: Esri France cartographic data, BRGM, completed.

Dans la classification en Local Climate Zone (LCZ) de Stewart et Oke (2012), cet espace correspond à la catégorie 6 (open low rise), c'est-àdire un espace ouvert caractérisé par la présence de constructions de faible hauteur (1 à 3 étages) et une proportion importante de jardins (sols perméables et recouverts de végétations basses et de quelques arbres).

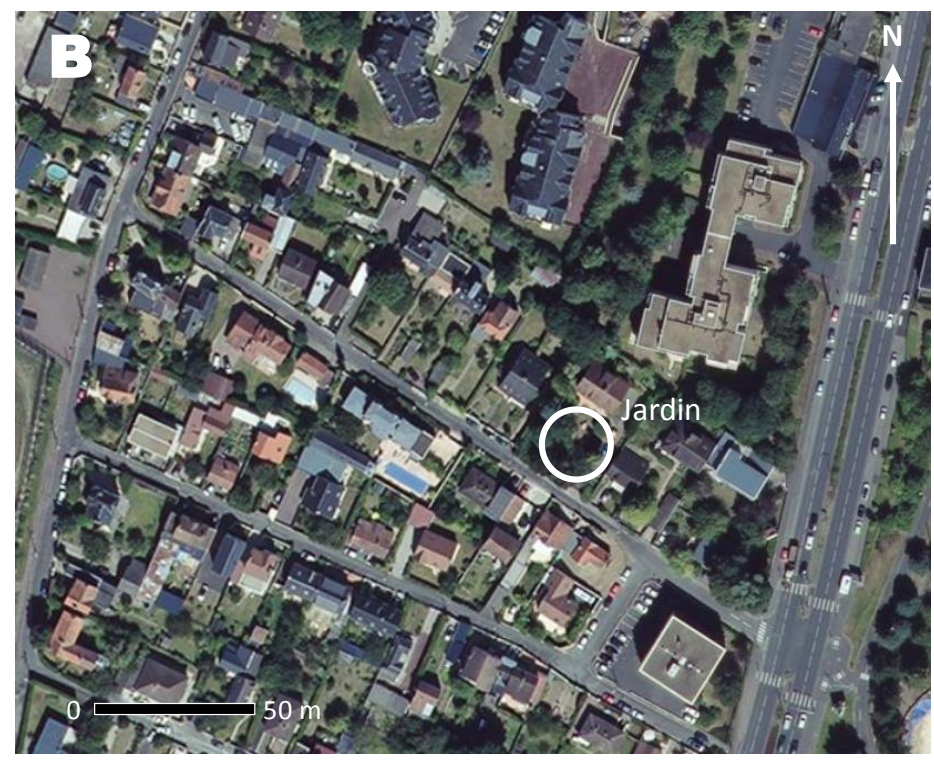

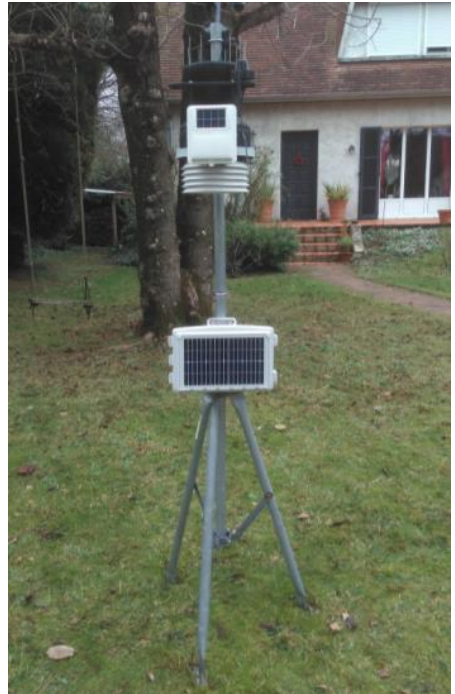

Photo. : Alexandre Follin (2019)

Figure 2. Caractéristiques géographiques des sites et instruments de mesure pour la station météorologique officielle de Caen-Carpiquet (en haut) et pour la station expérimentale Vantage Pro 2 en périphérie urbaine de Caen, allée des Merisiers (en bas). Sources : données cartographiques Esri France, BRGM, complétées. Geographical characteristics of the sites and measuring instruments for the official weather station of Caen-Carpiquet (top) and for the Vantage Pro 2 experimental station on the urban periphery of Caen, allée des Merisiers (bottom). Sources: Esri France cartographic data, BRGM, completed. 
Pour garantir la fiabilité des écarts de température observés entre les deux sites, la qualité de la station météorologique expérimentale, de type Vantage Pro 2 de Davis Instruments, a été validée préalablement par une campagne de mesures simultanées dans le parc instrumental de la station Météo France de Bernières-sur-Mer (pour des questions de sécurité, il n'est plus possible d'accéder sur le site de l'aéroport). Tous types de temps confondus, les écarts apparaissent insignifiants ${ }^{4}$ et autorisent donc une comparaison directe des valeurs entre les deux sites rural et périurbain. Les données exploitées dans cet article ont été enregistrées en période hivernale, entre le $1^{\mathrm{er}}$ janvier 2019 et le 15 mars 2019, soit une série de 74 jours consécutifs, constituée de 1776 échantillons horaires.

\section{Résultats et interprétation}

\subsection{Analyse des données au pas de temps journalier}

Sur la période étudiée, la périphérie ouest de Caen apparait légèrement plus chaude $\left(+0,40^{\circ} \mathrm{C}\right)$ que la campagne environnante (tableau 2). L'écart est plus sensible sur les valeurs minimales journalières $\left(+0,54^{\circ} \mathrm{C}\right)$ que sur les valeurs maximales $\left(+0,26^{\circ} \mathrm{C}\right)$, conformément à ce que l'on observe en général dans les grandes agglomérations où l'effet d'ICU est avant tout un phénomène nocturne, donc davantage visible sur les températures minimales que sur les maximales.

D'après les moyennes journalières des températures, l'îlot de chaleur périurbain (ICPU) est présent 8 jours sur 10 , mais son intensité est vraiment notable 1 jour sur 10 (écart supérieur à $+1^{\circ} \mathrm{C}$ dans $10,8 \%$ des cas). À ce seuil de dépassement, l'occurrence d'apparition est sensible sur les valeurs de température minimale journalière, avec près de 1 jour sur $4(24,3 \%)$, tandis que le phénomène n'est pratiquement pas existant sur les températures maximales $(1,4 \%)$.

Si le rouge pâle - révélateur d'un faible excédent de chaleur - domine la gamme chromatique du

\footnotetext{
${ }^{4}$ Sur une période de 26 jours consécutifs (du 6 au 31 octobre 2018), l'écart moyen de température a été de $0,02{ }^{\circ} \mathrm{C}$ entre la station officielle de Météo-France de
}

tableau 2, celle-ci est également parsemée de tâches bleu clair synonymes de la mise en place furtive d'un îlot de fraîcheur périurbain (IFPU). Cet IFPU est enregistré environ 1 jour sur 7 mais il reste de faible intensité puisqu'aucune valeur moyenne journalière n'atteint le seuil des $-1^{\circ} \mathrm{C}$ d'écart avec la campagne. Le phénomène apparait un peu plus franchement en dissociant les valeurs d'écart de températures minimales $(4,1 \%)$, des valeurs d'écart sur les températures maximales $(1,4 \%)$.

Notons que si 2 jours sur 3, l'écart ville/campagne reste positif simultanément pour les températures minimales et maximales (ICPU continu), seul 1 jour sur 25 propose un écart doublement négatif (IFPU continu). Enfin, environ 1 jour sur 5 on observe la succession d'une phase d'ICPU et d'IFPU dans la même journée, marque d'une forte cyclicité diurne/nocturne. Le nombre d'heures par jour avec des écarts thermiques significatifs (supérieurs à $+1^{\circ} \mathrm{C}$ ou inférieurs à $-1^{\circ} \mathrm{C}$ ) entre les deux stations permet d'apprécier plus concrètement la durée d'action de l'îlot de chaleur périurbain caennais « sensible » (ICPU+) et de son pendant négatif, l'îlot de fraîcheur périurbain « sensible » (IFPU+) (figure 3A) :

- l'ICPU+ se produit environ $10 \%$ du temps et son occurrence d'apparition est relativement bien répartie tout au long de la période d'étude, avec cependant quelques «jours sans » (notamment en janvier et début février) et de rares «pics » isolés (comme les $11 \mathrm{~h}$ observées le 28 janvier et les $8 \mathrm{~h}$ du 11 mars);

- l'IFPU+ représente un volume horaire plus réduit, de l'ordre de $4 \%$ du continuum temporel. Il se présente en 2 épisodes principaux durant le mois de février (numéros 1 et 2). Ces températures notablement et durablement plus basses en ville qu'à la campagne n'excluent pas dans la même journée l'établissement quasi systématique d'un ICPU marqué (ICPU+), traduisant des processus spécifiques qu'il conviendra d'élucider. Cette forte rythmicité journalière semble correspondre à des conditions claires et calmes (figure 3B).

Bernières-sur-Mer et notre station expérimentale Davis. Durant les 624 heures du test, $94 \%$ des observations présentent un écart compris entre $-0,2$ et $+0,2^{\circ} \mathrm{C}$. 
Tableau 2. Ecarts journaliers des températures minimales (TN), maximales (TX) et moyennes (TM) entre la périphérie

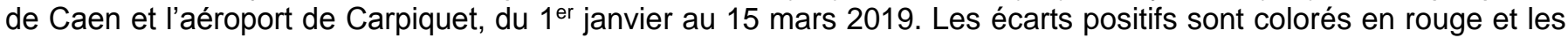
écarts négatifs en bleu. Les valeurs absolues supérieures à $1^{\circ} \mathrm{C}$ sont en gras. Sources : données Météo France de Carpiquet et station météorologique expérimentale périurbaine Vantage Pro 2. Daily differences in minimum (TN), maximum (TX) and average (TM) temperatures between the outskirts of Caen and Carpiquet airport, from January 1 to March 15, 2019. Positive deviations are colored in red and negative deviations in blue. Absolute values above $1^{\circ} \mathrm{C}$ are in bold. Sources: Météo France data from Carpiquet and Vantage Pro 2 peri-urban experimental weather station.

\begin{tabular}{|c|c|c|c|}
\hline \multirow[b]{3}{*}{1} & \multicolumn{3}{|c|}{ JANVIER } \\
\hline & dTN & $d T X$ & dTM \\
\hline & 0.4 & 0.1 & 0.3 \\
\hline 2 & 0.5 & 0.4 & 0.5 \\
\hline 3 & 0.1 & -0.6 & -0.3 \\
\hline 4 & -0.3 & 0.3 & 0.0 \\
\hline 5 & 0.9 & 0.5 & 0.7 \\
\hline 6 & 0.6 & 0.5 & 0.6 \\
\hline 7 & 1.6 & 0.4 & 1.0 \\
\hline 8 & 0.7 & 0.5 & 0.6 \\
\hline 9 & 1.1 & 0.9 & 1.0 \\
\hline 10 & 1.2 & 0.1 & 0.7 \\
\hline 11 & 0.6 & 0.1 & 0.4 \\
\hline 12 & 1.7 & 0.3 & 1.0 \\
\hline 13 & 0.5 & 0.5 & 0.5 \\
\hline 14 & 1.2 & 0.4 & 0.8 \\
\hline 15 & 1.0 & 0.1 & 0.6 \\
\hline 16 & 1.0 & 0.5 & 0.8 \\
\hline 17 & 2.1 & 0.3 & 1.2 \\
\hline 18 & 1.3 & 0.2 & 0.8 \\
\hline 19 & 1.1 & -0.5 & 0.3 \\
\hline 20 & 0.2 & 0.4 & 0.3 \\
\hline 21 & -1.0 & -0.3 & -0.7 \\
\hline 22 & 1.1 & 0.3 & 0.7 \\
\hline 23 & 0.7 & 0.6 & 0.7 \\
\hline 24 & 0.2 & 0.5 & 0.4 \\
\hline 25 & 1.4 & -0.1 & 0.7 \\
\hline 26 & 0.4 & 0.2 & 0.3 \\
\hline 27 & 0.0 & 0.5 & 0.3 \\
\hline 28 & 1.6 & 0.7 & 1.2 \\
\hline 29 & 1.0 & 0.7 & 0.9 \\
\hline 30 & 0.5 & -0.1 & 0.2 \\
\hline 31 & 0.4 & -1.1 & -0.4 \\
\hline
\end{tabular}

Figure 3. Nombre d'heures par jour avec un gradient thermique significatif entre la périphérie urbaine et la campagne environnante, du $1^{\text {er }}$ janvier au 15 mars 2019 (A). Vitesse du vent et fraction d'insolation (B). Sources: données Météo France de Carpiquet et station météorologique expérimentale périurbaine Vantage Pro 2. Number of hours per day with a significant thermal gradient between the urban periphery and the surrounding countryside, from January 1 to March 15, 2019 (A). Wind speed and insolation fraction (B). Sources: Météo France data from Carpiquet and the experimental periurban weather station Vantage Pro 2.

\begin{tabular}{|c|c|}
\hline \multicolumn{2}{|c|}{ FEVRIER } \\
\hline$\overline{d T X}$ & dTM \\
\hline-0.7 & -0.1 \\
\hline 0.4 & 0.2 \\
\hline-0.6 & 0.7 \\
\hline-0.1 & 0.4 \\
\hline 0.3 & 0.3 \\
\hline 0.1 & 0.3 \\
\hline 0.3 & 0.5 \\
\hline 0.2 & 0.4 \\
\hline 0.5 & 0.6 \\
\hline 0.4 & 0.3 \\
\hline 0.5 & 0.6 \\
\hline 0.3 & 0.4 \\
\hline-0.7 & -0.5 \\
\hline 0.7 & 0.6 \\
\hline 0.2 & -0.2 \\
\hline 0.6 & 0.0 \\
\hline 0.0 & -0.6 \\
\hline 1.0 & 0.5 \\
\hline-0.1 & 0.0 \\
\hline 0.0 & 0.5 \\
\hline-0.4 & -0.3 \\
\hline 0.1 & 1.1 \\
\hline 0.5 & 0.2 \\
\hline 0.4 & 1.0 \\
\hline 0.3 & 0.0 \\
\hline 0.5 & -0.1 \\
\hline 0.5 & 0.6 \\
\hline 0.5 & -0.6 \\
\hline
\end{tabular}

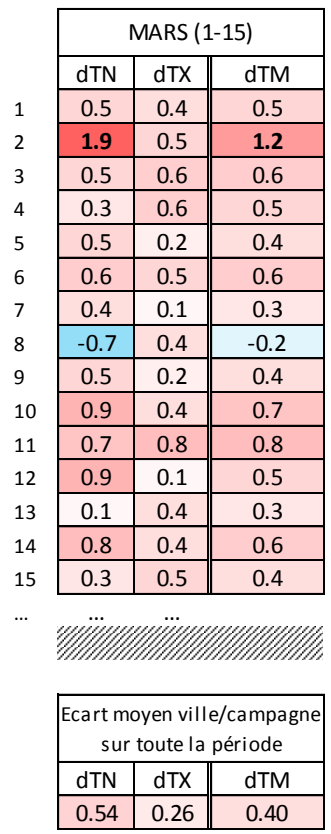

\begin{tabular}{|c|c||c|l|}
\hline \multicolumn{3}{|c|}{$\begin{array}{c}\text { Fréquence des écarts } \\
\text { ville / campagne }\end{array}$} \\
\cline { 1 - 2 } $\mathrm{TN}$ & $\mathrm{TX}$ & $\mathrm{TM}$ & classes \\
\hline $4.1 \%$ & $1.4 \%$ & $0.0 \%$ & $\mathrm{dT}<=-1^{\circ}$ \\
\hline $12.2 \%$ & $14.9 \%$ & $14.9 \%$ & $\mathrm{dT}\left[0^{\circ} \mathrm{C} ;-1^{\circ} \mathrm{C}[\right.$ \\
\hline $5.4 \%$ & $2.7 \%$ & $5.4 \%$ & $\mathrm{~d} \mathrm{~T}=0^{\circ} \mathrm{C}$ \\
\hline $54.1 \%$ & $79.7 \%$ & $68.9 \%$ & $\mathrm{dT}] 0^{\circ} \mathrm{C} ;+1^{\circ} \mathrm{C}[$ \\
\hline $24.3 \%$ & $1.4 \%$ & $10.8 \%$ & $\mathrm{dT}>=+1^{\circ} \mathrm{C}$ \\
\hline
\end{tabular}

A/ Nombre d'heures par jour d'écarts significatifs (supérieurs à $1^{\circ} \mathrm{C}$ )

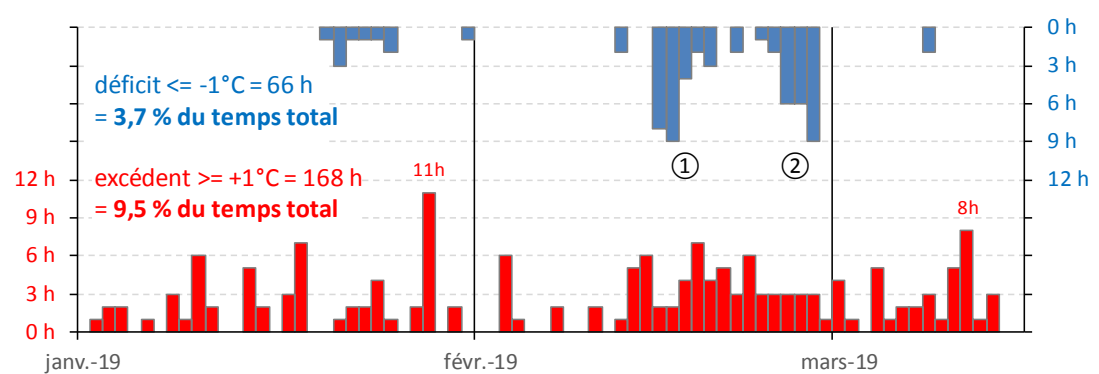

B/ Types de temps à l'aéroport de Caen Carpiquet

$\square$ Fraction d'insolation (\%) —Vitesse moyenne du vent $(\mathrm{m} / \mathrm{s})$

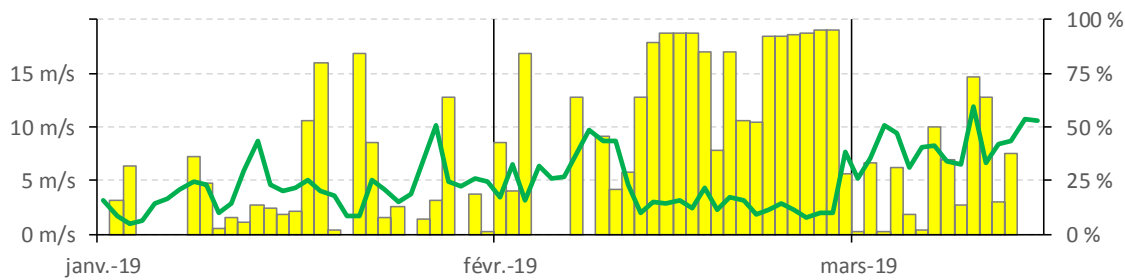




\subsection{Analyse des données au pas de temps horaire}

Les écarts thermiques horaires ville/campagne ${ }^{5}$ permettent de préciser les singularités de l'espace périurbain caennais, et notamment d'en apprécier plus concrètement les rythmes diurnes et nocturnes, jusqu'alors en grande partie masqués par l'analyse des seules valeurs minimales et maximales (tableau $3)$.

D'après les moyennes horaires établies du $1^{\mathrm{er}}$ janvier au 15 mars 2019, la légère surchauffe périurbaine s'accompagne d'un rythme journalier net. L'ICPU passe par un maximum d'intensité en fin de journée et en début de nuit (entre $+0,5$ et $+0,8^{\circ} \mathrm{C}$ de 16 à $20 \mathrm{~h} \mathrm{UTC}$ ), puis cet écart se réduit autour de valeurs faiblement positives tout au long de la nuit (entre $+0,1^{\circ} \mathrm{C}$ et $+0,3^{\circ} \mathrm{C}$ de $22 \mathrm{~h}$ à $8 \mathrm{~h}$ UTC). Il devient même neutre ou très faiblement négatif en début de matinée $\left(-0,1^{\circ} \mathrm{C}\right.$ à $9 \mathrm{~h}$ UTC), marque de l'apparition d'un IFPU.

Derrière ce rythme moyen se cachent des différences sensibles de comportement selon les types de temps (tableau 4), ainsi que le démontre ci- après la comparaison de deux séquences météorologiques contrastées entre la mi-février et début mars :

- par temps nuageux et venteux (ligne A du tableau 4), la périphérie ouest de Caen est constamment plus chaude que la campagne environnante (entre $+0,2^{\circ} \mathrm{C}$ et $+0,8^{\circ} \mathrm{C}$, pour une moyenne de $+0,5^{\circ} \mathrm{C}$ ). Le rythme journalier ressemble à celui observé sur l'ensemble de la période. Ce qui parait normal car ce type de temps perturbé domine à cette période de l'année. On note toutefois un léger lissage des écarts jour/nuit, vraisemblablement en raison de l'important brassage de l'air lié au vent et de l'absence d'échauffement différentiel significatif dû au faible rayonnement solaire diurne ;

- par temps clair et calme (ligne B du tableau 4), la périphérie ouest de Caen apparait tour à tour nettement plus chaude puis nettement plus froide que la campagne environnante. L'ICPU présent depuis la fin de matinée est particulièrement sensible au coucher du soleil $\left(+1,4^{\circ} \mathrm{C}\right.$ à $17 \mathrm{~h}$ UTC $)$, tandis que l'IFPU né en milieu de soirée atteint son maximum de froidure relative en début de matinée $\left(-1,4^{\circ} \mathrm{C}\right.$ à $9 \mathrm{~h}$ UTC $)$.

Tableau 3. Ecarts moyens de température horaire entre la périphérie ouest de Caen et l'aéroport, sur la totalité de la période. Les écarts positifs sont en rouge et les négatifs en bleu ; les valeurs absolues supérieures à $0,5^{\circ} \mathrm{C}$ sont en gras. Sources : données Météo France de Carpiquet et station météorologique expérimentale périurbaine Vantage Pro 2. Average hourly temperature differences between the western outskirts of Caen and the airport over the entire period. Positive deviations are in red and negative ones in blue; absolute values above $0.5^{\circ} \mathrm{C}$ are in bold. Sources: Météo France data from Carpiquet and Vantage Pro 2 peri-urban experimental weather station.

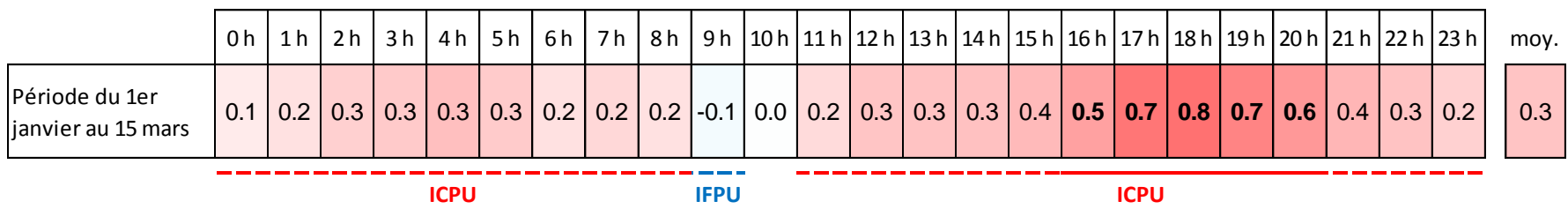

Il convient donc de s'intéresser plus spécialement à ces situations de «beau temps » puisque la présence d'un IFPU marqué (noté IFPU+) en fin de nuit et début de matinée est une caractéristique très originale car opposée à celle observée dans les grandes agglomérations où s'exprime le mieux l'ICU dans des situations synoptiques identiques. Ainsi, à titre de comparaison, en Île-de-France sur la période claire et calme évoquée précédemment, l'écart de

\footnotetext{
${ }^{5}$ L'écart moyen sur les 1776 relevés horaires est de
} $+0,32^{\circ} \mathrm{C}$, soit une valeur sensiblement équivalente à celle température entre la station rurale de Melun et le Jardin du Luxembourg au cœur de Paris était en moyenne de plus de $+3^{\circ} \mathrm{C}$ durant la nuit en faveur de la ville. Cette valeur d'ICU s'élevait même à plus de $+6^{\circ} \mathrm{C}$ avec la station amateur « hyper-urbaine » de St-Germain-des-Prés (tableau 5). Dans le même temps la périphérie urbaine ouest de Caen était plus froide que la campagne !

Ainsi, dans ces conditions claires et calmes a calculée à partir de la moyenne classique des températures minimales et maximales journalières $\left(+0,41^{\circ} \mathrm{C}\right)$. 
priori optimales pour la mise en place d'un fort ICU, comment expliquer la présence de cet îlot de fraîcheur nocturne dans la périphérie urbaine ouest caennaise et ce rythme atypique comparativement au modèle classique de climatologie urbaine ? Pour cela, il est nécessaire d'approfondir l'analyse des données en les examinant heure par heure dans leur continuum temporel réel et en prenant en compte les facteurs stationnels efficients.

Tableau 4. Ecarts moyens de température horaire entre la périphérie ouest de Caen et l'aéroport, par types de temps différents. Les écarts positifs sont en rouge et les négatifs en bleu ; les valeurs absolues supérieures à $0,5^{\circ} \mathrm{C}$ sont en gras. Sources : données Météo France de Carpiquet et station météorologique expérimentale périurbaine Vantage Pro 2. Average hourly temperature differences between the western outskirts of Caen and the airport, by different types of weather. Positive differences are in red and negative ones in blue; absolute values above $0.5^{\circ} \mathrm{C}$ are in bold. Sources: Météo France data from Carpiquet and the experimental peri-urban weather station Vantage Pro 2.

\begin{tabular}{|c|c|c|c|c|c|c|c|c|c|c|c|c|c|c|c|c|c|c|c|c|c|c|c|c|c|}
\hline & \multicolumn{8}{|c|}{ 2nde partie de NUIT } & \multicolumn{11}{|c|}{ JOUR (période de fin février à début mars) } & \multicolumn{5}{|c|}{ 1ère partie de NUIT } & \multirow[b]{2}{*}{ moy. } \\
\hline & $\mathrm{Oh}$ & $1 \mathrm{~h}$ & $2 \mathrm{~h}$ & $3 \mathrm{~h}$ & $4 \mathrm{~h}$ & $5 \mathrm{~h}$ & $6 \mathrm{~h}$ & $7 \mathrm{~h}$ & $8 \mathrm{~h}$ & $9 \mathrm{~h}$ & $10 \mathrm{~h}$ & $11 \mathrm{~h}$ & $12 \mathrm{~h}$ & $13 \mathrm{~h}$ & $14 \mathrm{~h}$ & $15 \mathrm{~h}$ & $16 \mathrm{~h}$ & $17 \mathrm{~h}$ & $18 \mathrm{~h}$ & $19 \mathrm{~h}$ & $20 \mathrm{~h}$ & $21 \mathrm{~h}$ & $22 \mathrm{~h}$ & $23 \mathrm{~h}$ & \\
\hline $\begin{array}{l}\text { A/ nuageux \& } \\
\text { venteux (1-6 mars) }\end{array}$ & 0.4 & 0.4 & 0.5 & 0.5 & 0.5 & 0.4 & 0.4 & 0.5 & 0.4 & 0.3 & 0.2 & 0.2 & 0.4 & 0.4 & 0.5 & 0.5 & 0.6 & 0.7 & 0.8 & 0.7 & 0.6 & 0.9 & 0.7 & 0.6 & 0.5 \\
\hline
\end{tabular}

\begin{tabular}{|c|c|c|c|c|c|c|c|c|c|c|c|c|c|c|c|c|c|c|c|c|c|c|c|c|c|}
\hline $\begin{array}{l}\text { B/ clair \& calme (13- } \\
16 \text { \& 22-27 fév.) }\end{array}$ & -0.3 & -0.2 & $-0.2 \mid$ & -0.1 & -0.1 & -0.1 & $-0.5 \mid$ & -0.3 & $-0.5 \mid$ & $|-1.4|$ & -0.8 & 0.3 & 0.4 & 0.2 & 0.1 & 0.2 & 0.7 & 1.4 & 1.3 & 0.6 & 0.2 & -0.2 & -0.3 & -0.3 & 0.0 \\
\hline
\end{tabular}

Tableau 5. Ecarts ville/campagne de la température horaire par temps clair et calme, à Caen et Paris sur la période 13-16 et 22-27 février 2019. Les écarts positifs sont en rouge et les négatifs en bleu. Sources : données Météo France de Paris-Jardin du Luxembourg (parc urbain), Melun (réf. rurale) et Caen-Carpiquet ; données Météociel de Paris StGermain-des-Prés (hyper centre); station météorologique expérimentale périurbaine caennaise Vantage Pro 2. City/country differences in hourly temperatures on clear and calm days in Caen and Paris over the period February 1316 and 22-27, 2019. Positive deviations are in red and negative ones in blue. Sources: Météo France data from ParisJardin du Luxembourg (urban park), Melun (rural ref.) and Caen-Carpiquet; Météociel data from Paris St-Germain-desPrés (hyper center); experimental weather station in the suburban area of Caen, Vantage Pro 2.

\begin{tabular}{|c|c|c|c|c|c|c|c|c|c|c|c|c|c|c|c|c|c|c|c|c|c|c|c|c|c|}
\hline & $\mathrm{Oh}$ & $1 \mathrm{~h}$ & $2 \mathrm{~h}$ & $3 \mathrm{~h}$ & $4 \mathrm{~h}$ & $5 \mathrm{~h}$ & $6 \mathrm{~h}$ & $7 \mathrm{~h}$ & $8 \mathrm{~h}$ & $9 \mathrm{~h}$ & $10 \mathrm{~h}$ & $11 \mathrm{~h}$ & $12 \mathrm{~h}$ & $13 \mathrm{~h}$ & $14 \mathrm{~h}$ & $15 \mathrm{~h}$ & $16 \mathrm{~h}$ & $17 \mathrm{~h}$ & $18 \mathrm{~h}$ & $19 \mathrm{~h}$ & $20 \mathrm{~h}$ & $21 \mathrm{~h}$ & $22 \mathrm{~h}$ & $23 \mathrm{~h}$ & noy \\
\hline & 3 & $\mid-0.2$ & $\mid-0.2$ & -0.1 & -0.1 & -0.1 & -0.5 & -0.3 & -0.5 & $|-1.4|$ & -0.8 & 0.3 & 0.4 & 0.2 & 0.1 & 0.2 & 0.7 & 1.4 & 1.3 & 0.6 & 0.2 & -0.2 & -0.3 & -0.3 & 0.0 \\
\hline & 3.2 & 3.0 & 3.1 & 2.9 & 3.2 & 3.2 & 3.2 & 3.1 & 2.3 & 3.6 & 3.2 & 2.8 & 2.5 & 2.6 & 2.6 & 2.6 & 2.4 & 1.5 & 2.1 & 2.1 & 2.4 & 2.4 & 2.4 & 2.9 & .. \\
\hline dT Paris (urbain ++) & 6.5 & 6.4 & 6.4 & 6.3 & 6.3 & 6.3 & 6.1 & 6.0 & 4.5 & 3.2 & 2.4 & 1.7 & 1.3 & 1.3 & 1.1 & 1.4 & 2.3 & 3.8 & 5.4 & 5.9 & 6.2 & 6.2 & 6.6 & 6.9 & 4.6 \\
\hline
\end{tabular}

\subsection{Une clé d'explication aux singularités thermiques péri-urbaines caennaises: l'association des facteurs stationnels et des types de temps}

Parmi les 74 jours de la série analysée, trois séquences de deux jours consécutifs avec des types de temps contrastés ont été extraits pour caractériser puis expliquer les causes des principaux écarts de température entre la station rurale de référence de Caen-Carpiquet et l'espace périurbain de l'allée des Merisiers, à l'ouest de la ville (figure 4). La présentation des résultats sous la forme d'un continuum temporel au pas de temps horaire permet dans un premier temps de confirmer certaines hypothèses sur le rôle des types de temps dans la formation de l'ICPU périurbain caennais (cas A et B), et de saisir dans un second temps l'origine des mécanismes plus subtils conduisant à l'apparition paradoxale d'un IFPU sous certaines conditions d'échelle synoptique (cas C).

3.3.1. Par temps plus ou moins nuageux et/ou plus ou moins venteux: homogénéisation thermique ville/campagne

Dans ces conditions les plus fréquentes en climat océanique (colonnes A et B), les mécanismes de 
formation d'un climat local différencié sont faiblement actifs, quel que soit la dimension de l'agglomération. À Caen, l'écart de température entre l'allée des Merisiers et l'aéroport est alors relativement stable tout au long de la journée, limité à quelques dixièmes en faveur de la ville. Cette valeur moyenne tournant autour de $+0,5^{\circ} \mathrm{C}$ pourrait constituer le signal de fond de l'îlot de chaleur périurbain caennais?

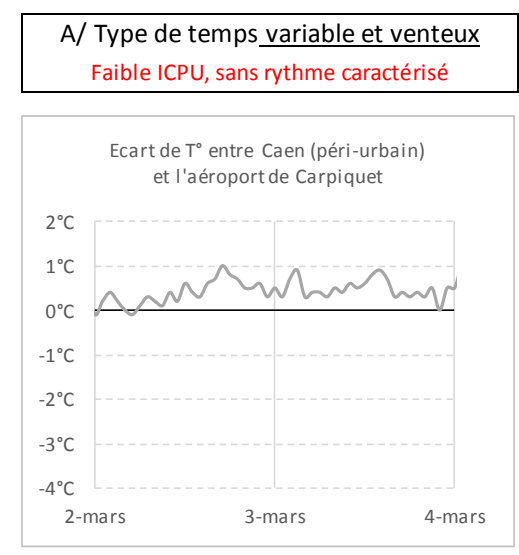

Paramètres météorologiques associés
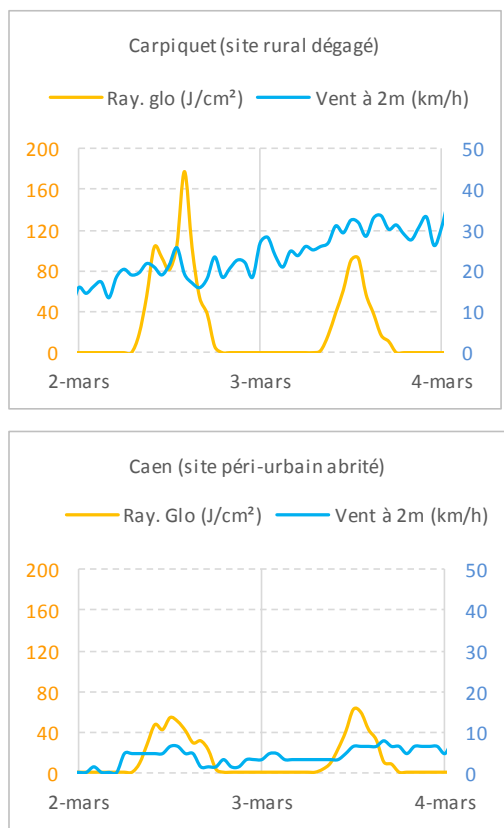

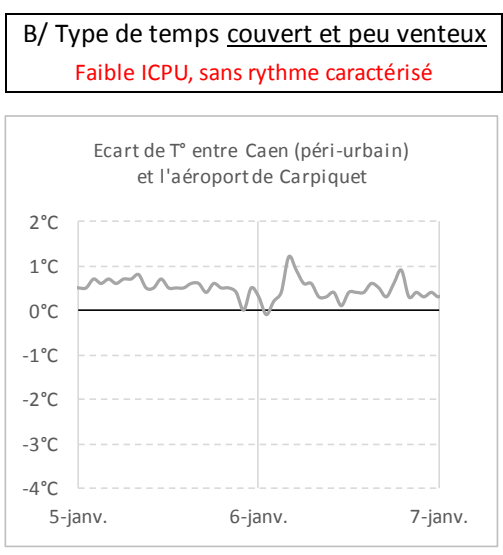

Paramètres météorologiques associés
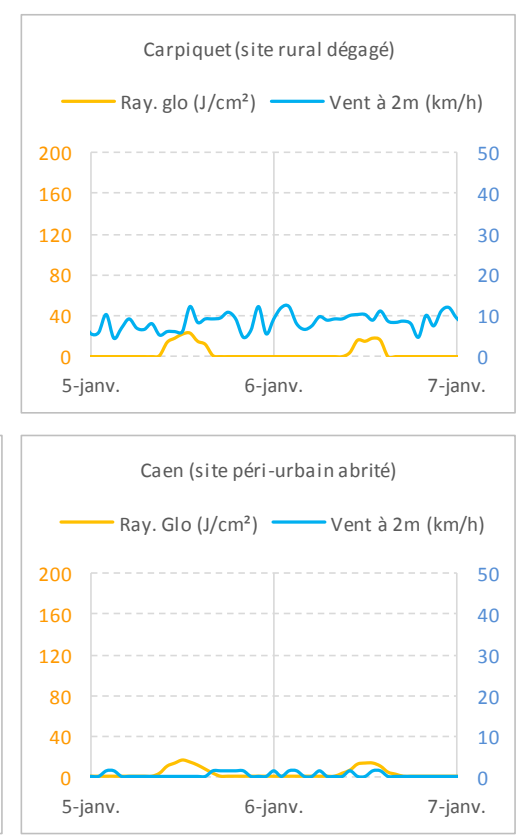

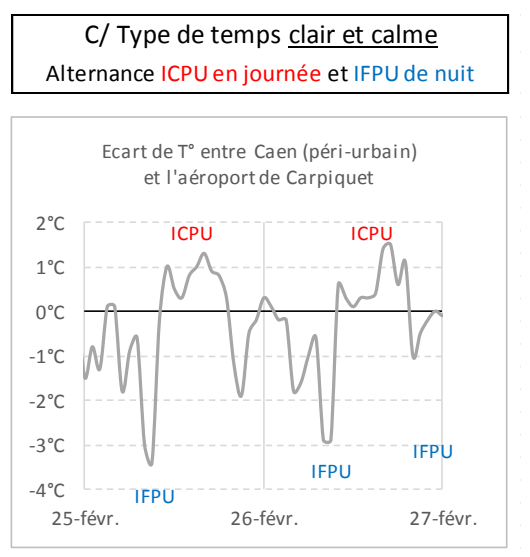

Paramètres météorologiques associés
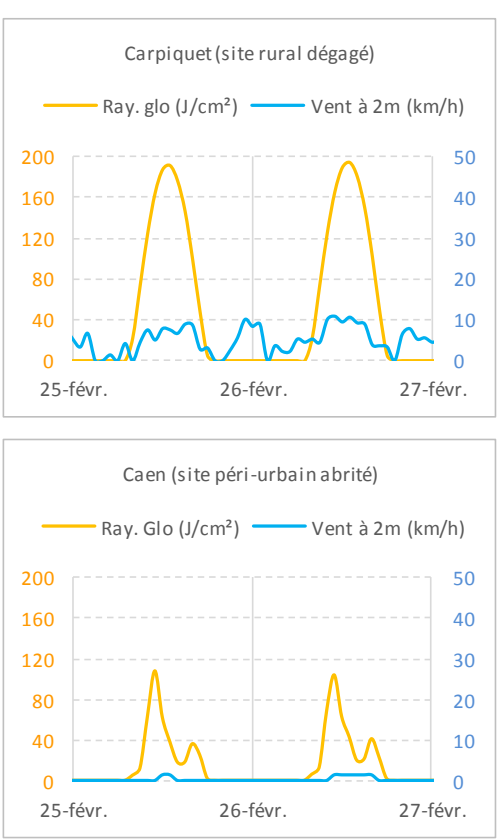

Figure 4. Influence des types de temps sur le gradient thermique entre la périphérie urbaine de Caen et l'aéroport de Carpiquet. Sources : données Météo France de Carpiquet et station météorologique expérimentale périurbaine Vantage Pro 2. Influence of weather types on the thermal gradient between the urban periphery of Caen and Carpiquet airport. Sources: Météo France data from Carpiquet and Vantage Pro 2 peri-urban experimental weather station.

\subsubsection{Par temps clair et calme : des situations rares aux conséquences inattendues}

De nuit, par temps clair et calme (colonne C), le secteur périurbain à l'ouest de Caen se distingue par une température plus basse que sur l'aéroport (fréquemment 1 à $3^{\circ} \mathrm{C}$ de moins). Ce fait atypique apparait difficilement dans les statistiques car ce type de temps est peu fréquent à l'échelle de l'année
$(7,9 \%)$ et encore moins en hiver $(4,3 \%)$ (rappel tableau 1). Dans la journée la situation s'inverse et la ville est alors plus chaude que la campagne durant une dizaine d'heures consécutives. La figure 5 permet de mieux visualiser ce rythme alterné d'apparition d'un îlot de fraîcheur périurbain nocturne (IFPUn) et d'un îlot de chaleur périurbain diurne (ICPUd). 

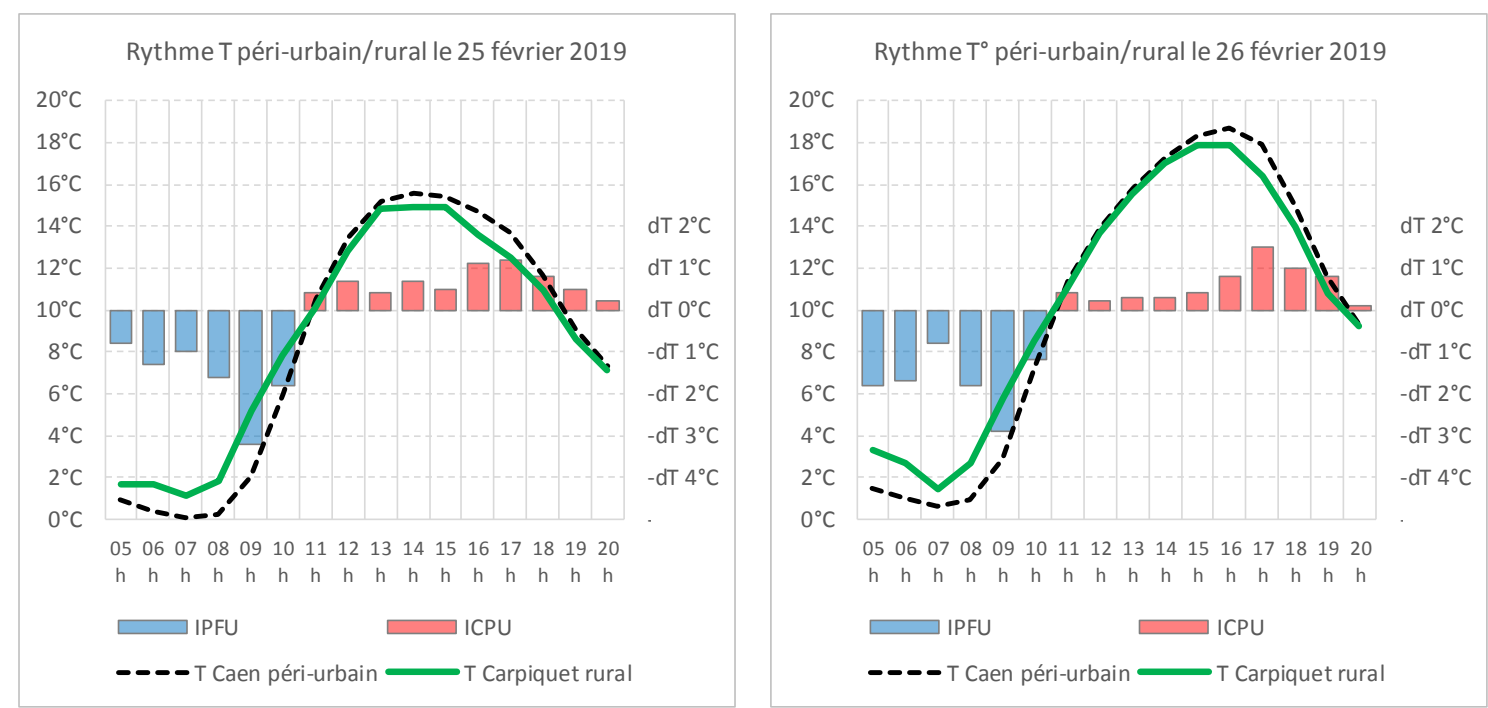

Figure 5. Comparaison du cycle diurne des températures en périphérie urbaine ouest de Caen et à l'aéroport de Carpiquet. Îlot de fraîcheur périurbain nocturne (IFPUn) et îlot de chaleur périurbain diurne (ICPUd). Sources : données Météo France de Carpiquet et station météorologique expérimentale périurbaine Vantage Pro 2. Comparison of the diurnal temperature cycle in the western urban periphery of Caen and at Carpiquet airport. Night-time peri-urban cool island (nPUCl) and daytime peri-urban heat island (dPUHI). Sources: Météo France data from Carpiquet and the experimental peri-urban weather station Vantage Pro 2.

\subsubsection{Des pistes d'explications...}

Les facteurs et processus à l'origine de cette anomalie au schéma habituel de l'ICU par temps clair et calme tiennent tout à la fois aux caractéristiques géographiques de l'espace périurbain considéré, que de celles de la station rurale de référence (ici en parfait accord avec les normes internationales pour l'implantation d'une station météorologique) :

Le premier élément à considérer est la morphologie où est installée la station de référence urbaine, rue des Merisiers. Ce quartier à l'ouest de Caen est essentiellement résidentiel et de ce fait présente peu de grandes surfaces minérales et de gros volumes bâtis, ce qui contribue à limiter l'emmagasinement «anthropique » de chaleur en journée sous l'action du soleil. Par ailleurs, cet espace présente un déficit important de rayonnement solaire (figure 4). Les constructions bien que d'élévation modeste - créent à cette saison de grands cônes d'ombre qui limitent le pourcentage de surfaces soumises au rayonnement direct du soleil et contribuent de ce fait à maintenir de la fraîcheur. Ce frein au réchauffement diurne est renforcé par la présence de végétation qui intercepte également le soleil et de la proportion non négligeable de sols naturels (jardins) dont la capacité d'absorption calorifique est moindre que celle des matériaux urbains. Mais ces caractéristiques ne sont pas suffisantes à elles seules pour comprendre l'anomalie thermique nocturne (IFPU à la place attendue d'un ICPU) qui caractérise ce secteur par temps clair et calme.

Pour comprendre l'origine de cet air plus froid en ville qu'à la campagne, il faut comparer les conditions de refroidissement nocturne par rayonnement pour les deux sites de références :

- À Caen, Allée des Merisiers, les constructions et la végétation forment des obstacles pour le vent (figure 4). Celui-ci est totalement calme, facilitant la formation d'une inversion de température nocturne près du sol quand le ciel est dégagé. Le relief plat et la présence de haies végétales rapprochées bloquent tout mouvement gravitaire de l'air froid plus dense. Le froid peut donc s'accentuer et la couche s'épaissir au fil des heures. Le refroidissement est d'autant plus rapide et efficace que la réserve «anthropique » de chaleur est réduite.

- Dans le même temps, à l'aéroport de Carpiquet, le ciel est également totalement clair mais un très léger vent persiste sur ce plateau dépourvu de tout obstacle, ce qui freine le refroidissement dans les très basses couches. 
Cet IFPU atteint son maximum d'intensité en début de matinée (environ $-3^{\circ} \mathrm{C}$ vers 9 h UTC). Dans les premières heures de la journée, la station périurbaine reste alors dans une ambiance froide, calme et à l'abri des rayons directs du soleil encore trop bas (effet d'écran). Tandis que sur le site de l'aéroport, l'air déjà moins froid formé près du sol durant la nuit est rapidement dissipé par les effets du soleil qui brille « librement » et par le vent qui se renforce simultanément, ce qui contribue à creuser temporairement un écart substantiel avec la ville, encore plongée en quelque sorte dans son « microlac » d'air froid.

Mais cette IFPU est fragile et il cesse ensuite brusquement vers $11 \mathrm{~h}$ UTC, en relation avec les premières petites turbulences urbaines, nées des différences d'échauffement entre espaces éclairés et ombragés, qui détruit rapidement la poche froide jusqu'alors emprisonnée près du sol. L'ICPU se met alors immédiatement en place pour tout le reste de la journée en raison du confinement de l'air en ville qui permet aux températures d'augmenter plus vite que sur la campagne où le brasage de l'air freine le réchauffement. L'excédent thermique urbain diurne persiste jusque dans les premières heures de la nuit (20 h UTC), c'est-à-dire tant que la chaleur emmagasinée dans les matériaux de construction n'est pas cédée totalement à l'atmosphère. Cette courte phase de restitution différencie la périphérie urbaine de Caen des grandes agglomérations où le phénomène se produit tout au long de la nuit en raison du stock de chaleur plus important (à l'origine d'un fort ICU nocturne). De plus, l'agglomération caennaise est certainement trop petite pour que le refroidissement nocturne périurbain soit compensé par la diffusion de chaleur à partir du cœur de ville (au contraire de Paris, par exemple, où une partie de l'ICU peut même se reporter plusieurs kilomètres sous le vent de la ville (Escourrou, 1991 ; Cantat, 2004).

Par ailleurs, à échelle plus fine, la composition de ce quartier périurbain de Caen est défavorable à l'accumulation de chaleur dans la journée car il est constitué de maisons individuelles et de petits immeubles collectifs largement pourvus en espaces verts qui sont le siège en présence de soleil d'une consommation d'énergie non négligeable par évapotranspiration limitant la conversion du rayonnement solaire en chaleur sensible (cf. catégorie 6 «open low rise» de la classification LCZ).

\section{Discussion et conclusion}

$\mathrm{Au}$ terme de cette analyse exploratoire, l'explication des faits dans toute leur diversité et leur complexité renvoie aux questions classiques en géographie de représentativité, d'échelles et de limites, comme nous allons le préciser ci-dessous à partir de nos observations.

Ainsi, l'agglomération caennaise propose un espace périurbain légèrement plus chaud que le milieu rural environnant $\left(+0,4^{\circ} \mathrm{C}\right)$, ce en quoi il s'insère dans une logique spatiale conforme au modèle de climatologie urbaine, dans le cadre d'une agglomération de moyenne dimension. Vu à travers le filtre des moyennes journalières, le phénomène d'îlot de chaleur apparait également conforme à la schématisation usuelle avec un excédent plus marqué pour les températures minimales $\left(+0,54^{\circ} \mathrm{C}\right)$ que sur les maximales $\left(+0,26^{\circ} \mathrm{C}\right)$

Mais au-delà de cette réalité «moyenne », une analyse du climat vécu (au pas de temps court de la succession des heures et des journées) met en évidence une forte singularité de comportement thermique pour cet espace périurbain. Lors des séquences atmosphériques claires et calmes, apparaît un îlot de fraîcheur nocturne en lieu et place théoriquement d'un îlot de chaleur. Les interactions entre les caractéristiques physiques des surfaces à très fine échelle et la vitesse du vent semblent alors déterminantes. La réponse à cette anomalie majeure fait davantage appel au poids des facteurs stationnels (micro-échelle) qu'à une position géographique à mi-chemin entre le cœur de ville et la campagne (méso-échelle). La réalité des faits mesurés ne correspond donc pas nécessairement à la figuration classique de l'îlot de chaleur sous la forme d'un dôme régulier s'atténuant progressivement du centre de l'agglomération vers sa périphérie rurale.

Pour asseoir climatologiquement les conclusions de cette étude, il conviendrait d'élargir dans le temps et dans l'espace nos observations en nous interrogeant en amont sur plusieurs points : 
1) la représentativité spatiale de l'emplacement de la station expérimentale

L'allée des Merisiers est représentative du périurbain résidentiel. Pour caractériser plus spécifiquement l'influence de l'urbanisation sur le climat à Caen il serait souhaitable d'instrumenter un espace vert, plan et relativement dégagé situé en plein centre-ville. Une fois l'endroit idéal repéré (exemple ci-après de la Place de la Résistance, figure 6), se posent alors d'autres contraintes liées d'une part à la sécurité du matériel et d'autre part à l'autorisation de disposer de l'espace public. Des démarches en ce sens vont être entreprises prochainement auprès de la Mairie de Caen.

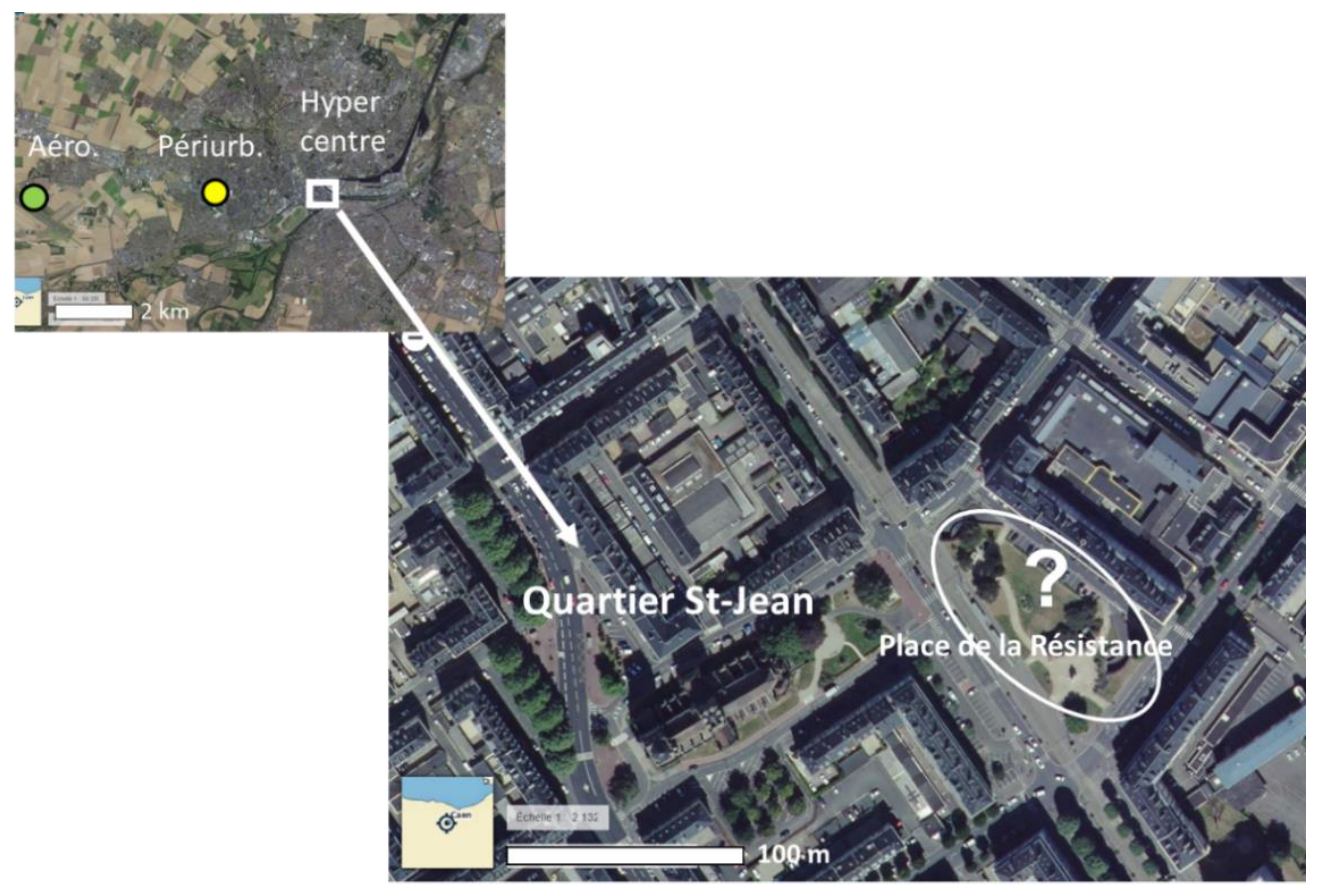

Figure 6. Exemple d'emplacement potentiel pour l'installation d'une station météorologique de référence urbaine à Caen. Source: données cartographiques Esri France, BRGM, complété. Example of a potential location for the installation of an urban reference weather station in Caen. Source: Esri France cartographic data, BRGM, completed.

Pour avoir une image plus complète du climat urbain caennais, en dehors de l'hypercentre, il serait également souhaitable d'instrumenter plusieurs autres sites représentatifs de la diversité de l'espace urbain, par exemple en s'aidant de la typologie Local Climate Zone établie par Stewart et Oke (2012). A la manière de ce qui a été réalisé sur la Métropole de Rennes (Foissard, 2015) et de Dijon (Richard et al., 2018) par exemple.

2) la représentativité temporelle de la période d'analyse :

L'« identité thermique » de la ville de Caen ne peut s'établir à partir de la seule connaissance des écarts ville/campagne mesurés sur une période de deux mois et demi. Pour avoir une idée plus précise des spécificités urbaines, il faudrait disposer a minima d'un panel de mesures correspondant aux différentes saisons et leurs différents types de temps. En effet, le différentiel urbain/rural varie de façon cyclique sous la dépendance de données astronomiques immuables (comme la hauteur du soleil et la durée du jour qui règlent l'apport énergétique incident), de façon saisonnière mais variable d'une année à l'autre (en lien avec les stades de la végétation et l'état des sols via les flux évapotranspiratoires et les effets d'ombre et de lumière) et enfin de façon beaucoup plus aléatoire selon les types de temps.

À titre d'exemple, notons que la fréquence hivernale moyenne de l'IFPU est certainement plus faible que celle calculée sur les 74 jours analysés ici car les conditions synoptiques propices à un temps clair et calme sur la région ont été anormalement durables début 2019 (tableau 6) : 12,2 \% des journées affectées par ce type de temps contre seulement $4,3 \%$ en moyenne sur la période 19812010. 
Tableau 6. Combinaisons des éléments météorologiques favorables à l'expression des facteurs géographiques locaux

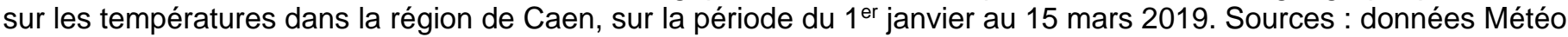
France de Caen-Carpiquet et des types de circulation atmosphérique selon la classification de Hess \& Brezowsky. Combinations of weather elements favourable to the expression of local geographical factors on temperatures in the Caen region, over the period from January 1 to March 15, 2019. Sources: Météo France data for Caen-Carpiquet and atmospheric circulation patterns according to the Hess \& Brezowsky classification.

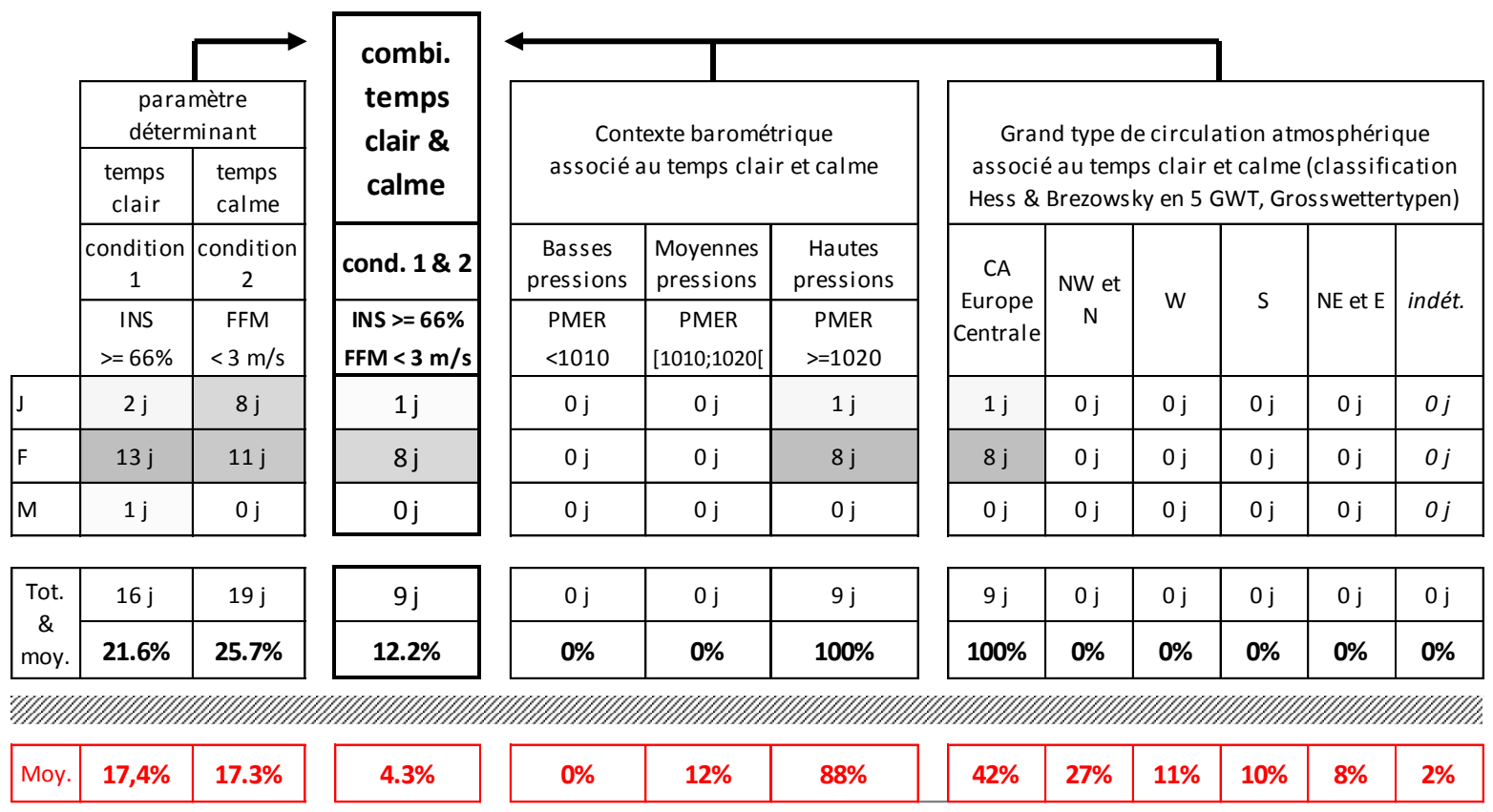

ci-dessus en rouge, rappel des valeurs sur la Normale 1981-2010 pour la période du 1er janvier au 15 mars

Au final, pour préciser les caractères thermiques singuliers de la ville de Caen et affiner les hypothèses explicatives proposées, il conviendrait de $(i)$ prolonger ce comparatif sur les autres saisons de l'année, (ii) disposer d'une référence au cœur de l'agglomération et (iii) instrumenter les autres types d'urbanisation représentatifs de l'espace géographique, selon une classification LCZ (Stewart et Oke, 2012).

\section{Références}

Besancenot J.-P., 2004. Une vague de chaleur meurtrière : les enseignements de l'été 2003 en France. Géoconfluences DESCO - ENS LSH, Brève, 6. http://geoconfluences.enslyon.fr/

Cantat O., 2004. L'îlot de chaleur urbain parisien selon les 'types de temps'. Norois, 191, 75-10.

Carrega P., 1992. Topoclimatologie et habitat. Thèse Doctorat d'Etat, publiée en 1994 dans la Revue d'Analyse Spatiale, Nice, 35-36, $408 \mathrm{p}$.

Carrega P., 2013. Le climat urbain de Nice en milieu géographique contrasté : synthèse par approche inductive. Climatologie, 10, 9-34.

Dubreuil V., Quénol H., Foissard X. et Planchon O., 2011. Climatologie urbaine et îlot de chaleur urbain à Rennes. Ville et biodiversité, Clergeau P. (dir.), Presses Universitaires de Rennes, 137-159.

Escourrou G., 1978. Climats et types de temps en Normandie. Thèse de doctorat d'Etat de géographie, Champion, Paris, 1081 et $525 \mathrm{p}$.

Foissard X., 2015. L'îlot de chaleur urbain et le changement climatique: application à l'agglomération rennaise. Thèse, Géographie, Université Rennes 2, 248 p.

Foissard X., Dubreuil V., Quénol H, 2019. Defining scales of the land use effect to map the urban heat island in a mid-size European city: Rennes (France). Urban Climate, 29, 100490.

Hess P., Brezowsky H., 1952. Katalog der Grosswetterlagen Europas. Bibliothek des Deutschen Wetterdienstes in der USZone, 33, $39 \mathrm{p}$.

Joly D., Brossard T., Cardot H., Cavailhes J., Hilal M. et Wavreskyjoly P., 2010. Les types de climats en France, une construction spatiale. Cybergeo : European Journal of Geography [En ligne], Cartographie, Imagerie, SIG, document 501, mis en ligne le 18 juin 2010. URL: http://cybergeo.revues.org/23155 ; 10.4000/cybergeo. 23155

IAU Île-de-France, 2010. Les îlots de chaleur urbains. L'adaptation de la ville aux chaleurs urbaines. 78 p., http://www.iau-idf.fr/fileadmin/Etudes/etude 761

INVS, 2003. Impact sanitaire de la vague de chaleur en France survenue en août 2003. https://www.santepubliquefrance.fr/ Kastendeutch P., Najjar G., Lacarrere P., Colin J., 2010. 
Modélisation de l'ICU à Strasbourg. Climatologie, 7, 21-37. Lauffenburger M., Claverie R., Bouyer J., Combas P., Reveilleaux C., 2014. Mise en place d'une méthode d'estimation du flux d'évapotranspiration en milieu urbain pour l'étude du bilan énergétique de quartier de l'agglomération de Nancy. Actes des Journées de climatologie de la commission Climat et Société du CNFG, Caen, 93-110. Leroy M., 1999. Classification d'un site. Notes techniques, $N^{\circ} 35$, DSO, Météo-France, $12 \mathrm{p}$.

Pedelaborde P., 1958. Le climat du Bassin parisien : essai d'une méthode rationnelle de climatologie physique. Paris, Génin, 539 p.
Richard Y., Emery J., Dudek J., Pergaud J., Chateau Smith C., Zito S., Rega M., Vairet T., Castel T., Thevenin T., Pohl B., 2018. How relevant are Local Climate Zones and Urban Climate Zones for urban climate research? Dijon (France) as a case study. Urban Climatology, 258-274.

Stewart I. D., Oke T. R., 2012. Local climate zones for urban temperature studies. Bull. Amer. Meteor. Soc., 93(12), 18791900.

Sites internet :

https://www.insee.fr/fr/statistiques

Citation de l'article : Cantat O. et Follin A., 2020. Les singularités thermiques d'un espace périurbain dans une agglomération de dimension moyenne : le cas de Caen durant l'hiver 2018/2019. Climatologie, 17, 3. 